\title{
EFFECT OF DIETARY SUPPLEMENTATION WITH SOYBEAN ON REPRODUCTIVE HORMONES OF MALE WISTAR RATS
}

\author{
Anisah, Yahya ${ }^{1}$, $*$ Mohammed, I.Y. ${ }^{2}$ and Jibril, M. El-Bashir ${ }^{3}$ \\ 1. Department of Obstetrics \& Gynaecology, College of Medical Sciences, Ahmadu Bello \\ University/Ahmadu Bello University Teaching Hospital, Zaria, Kaduna State. \\ 2. Department of Chemical Pathology, Bayero University Kano, Kano State. \\ 3. Department of Chemical Pathology, College of Medical Sciences, Ahmadu Bello \\ University/Ahmadu Bello University Teaching Hospital, Zaria, Kaduna State. \\ *Corresponding Author; iymohammed.cpat@buk.edu.ng
}

ABSTRACT

Dietary soybean has been shown to have variable effects on the reproductive system of male rodents, non-human primates and humans. These effects can affect subsequent fertility. Soybean intake in men has been advocated due to its protective effect in the prevention of prostate cancer, cardiovascular disease and osteo-arthritis. However, the protection it offers in the prevention of prostatic cancer by altering the levels of male reproductive hormones may lead to infertility. This study aimed at determining the effect of dietary supplementation with soybean on reproductive hormones of male Wistar rats. The study was a randomised controlled trial. Adult male Wistar rats were randomly assigned to a control group and two study groups. The rats in the control group were fed on soy-free diet while rats in Group 1 and Group 2 of the study were fed on $20 \%$ and $50 \%$ soybean supplemented diet for four weeks. Serum concentration of FSH, LH and Testosterone were estimated. Data obtained was analysed using statistical package for social sciences (SPSS) version 20.0.There was no statistically significant difference ( $p<$ $0.05)$ in the level of FSH $(p=0.783), L H(p=0.815)$ and Testosterone $(p=0.330)$ between the study and control groups. Dietary supplementation with of soybean has no effect on the level of FSH, LH and Testosterone in male Wistar rats and therefore may not affect their reproductive function.

Keywords: Soybean,FSH, LH, Testosterone, Wistar Rats

\section{INTRODUCTION}

Soybean is a species of leguminous plants that belong to the Fabaceae group of plants and is a rich dietary source of bioactive phytoestrogen. Phytoestrogens are subdivided into isoflavones, flavones, lignans and coumestans. The phytoestrogen content of soybean belongs to the isoflavone group of phytochemicals. Each gram of soybean contains approximately $3.5 \mathrm{mg}$ of isoflavone (Massina et. al., 2006). Genistein is the main isoflavane of soybean. Phytoestrogen is a natural selective oestrogen receptor modulator (Weber et. al., 2001). Phytoestrogens occur naturally in an inactive glycosidic form but are hydrolyzed by intestinal bacteria to form biologically active aglycone products that can be absorbed into the blood stream. It has all the physiological and physiochemical characteristics of oestrogen (Moderasi et al., 2011; Crouse et. al., 1999).It is non-steroidal and has diphenolic structure. It has weak oestrogenic activity being able to bind oestrogen receptor a with an affinity 100-1000 times lower than estradiol
(Adlercneutz et. al., 2000)but bind strongly to membrane oestrogen receptor to exert nongenomic actions potentially deleterious to male fertility (Serag El Din et. al., 2011).

Phytoestrogens have been reported to have a protective effect in prevention of development of cardiovascular diseases, osteoporosis and hormone-dependent cancers like breast and prostate cancers (Adlercneutz et. al.,2000; Knight et. al., 1996; Adlercneutzand Mazzur, 1997). The anticancer effect of phytoestrogens is associated with several possible mechanisms. They can inhibit tyrosine kinase, growth factors, and DNA topoisomerase and steroidogenic enzymes. They also can act as antioxidant and anti-angiogenic agents. All these properties prevent carcinogenesis (Knight et. al., 1996; Price and Fenwick, 1995; Setchell 1998).

Despite the possible enormous health benefits that can be derived from dietary intake of soybean, it has been implicated as a possible cause of male infertility (Fraser 2006, Atanassoun et. al., 2000). Soybean has been 
BAJOPAS Volume 13 Number 2, December, 2020 associated with impaired development of reproductive organs following intrauterine exposure and abnormal spermatogenesis and low sperm concentration following exposure in adult males leading to male factor infertility (Hamilton-reeves et. al., 2007). Some studies have demonstrated no effect on concentration of serum testosterone, follicle stimulating hormone, luteinizing hormone, and serum concentration of testosterone in men (Liang and McGrath, 2001; Perry et. al., 2007).

On the other hand, some studies () reported some adverse effects following dietary ingestion of soybean on the reproductive physiology in both human and animal models of research. These adverse effects include decrease in serum free testosterone, lower sperm concentration, increase in serum estradiol (E2) and oestrone (E1) and adverse effect on spermatogenesis (Songet. al.,1999; Lund et. al.,2004; Goodin et. al.,2007; Dillingham et. al., 2005).

This study sought to determine the effect of dietary supplementation with soybean on $\mathrm{FSH}$, $\mathrm{LH}$ and Testosterone levels in male Wistar rats.

\section{MATERIALS AND METHODS}

\section{Study Design}

The study was a randomised controlled trial. The control group was given soy free diet. The experiment groups were given different concentration of soy-based diet as explained under randomization section below. It was conducted at the Department of Human Physiology Bayero University, Kano. The laboratory tests were conducted at the Department of Chemical Pathology, Aminu Kano Teaching Hospital, Kano.

\section{Ethical Consideration:}

Approval to conduct the research was obtained from the Research and Ethics committee of the College of Health Sciences, Bayero University Kano (BUK/CHS/REC/01/41). Ethics of use of animals for research was adhered to.

\section{Study Animals}

A total of 30 Inbred male post pubertal Wistar rats (three months old) weighing $120 \mathrm{~g} \pm 20 \mathrm{~g}$ were obtained from the animal house of Bayero University, Kano. They were kept in plastic cages with solid floor, saw dust bedding/nesting, at a room temperature of $25-27^{\circ} \mathrm{C}$ and $12 / 12$ hours light/dark cycle to acclimatize over a period of 2 weeks.

\section{Randomization}

After the period of adaptation, the animals were randomly assigned (using random number table) to three groups. Each group had a total of 10 rats. Group 1 was the control group and Groups 2 and 3 were the experimental groups. Each group had two cages with five rats per cage.
The cages were labelled 1 to 3 indicating the group. The rats in each cage were numbered based on cage number and number in the cage. Thus, the rats were labelled 1.1 to 1.10 in group $1,2.1$ to 2.10 in group 2 and 3.1 to 3.10 in group 3.

\section{Formulation of Study Diet}

Soybean was obtained from a local market in Zaria, Kaduna state. The soybean was fermented in water for 12 hours to break the oligosaccharides within it. It was then cooked at $120^{\circ} \mathrm{Cfor} 18-20$ minutes. This will decrease the anti-nutrient content within it which includes trypsin inhibitors, phytin, lectins, saponins and haemagglutinins. It was then dried and ground into powder.

Twenty percent Soybean supplemented diet was prepared by mixing $20 \mathrm{~g}$ of the grounded soyabeans with $80 \mathrm{~g}$ of Growers mash while $50 \%$ soybean supplemented diet was prepared by mixing $50 \mathrm{~g}$ of the grounded soybean with $50 \mathrm{~g}$ of Growers mash.

\section{Animal Feeding}

Rats in group 1 (control) were fed on Growers mash and given water ad libitum while rats in the study group were fed on twenty percent (group 2) and fifty percent (group 3) soybean supplemented diet prepared as stated above and given water ad libitum. Rats in each cage (5) were given $100 \mathrm{~g}$ of the respective feed per day. The animals were fed on these diets for 6 weeks. The period represented at least four reproductive cycles.

\section{Blood Sample Collection and Processing}

After the period of experiment, trunk blood was obtained from all animals. A sterile blade was used to make an incision on the trunk. The blood was collected directly into plain bottles. The blood was spun at 1000 rpm for 5 minutes to separate the serum from the cells. The serum was stored in plain bottles labelled with rats' identification number at $-20^{\circ} \mathrm{C}$ and used for the test.

\section{Assay Technique}

LH, FSH test kit (obtained from Wuhan Fine Biotech Co., Ltd. Wuhan, China) principle is based on Competitive Enzyme Linked Immunosorbent Assay technique (ELISA) detection method. The microtiter plate provided in this kit has been pre-coated with target. During the reaction, target in the sample or standard competes with a fixed amount of target on the solid phase supporter for sites on the Biotinylated Detection Antibody specific to target. Excess conjugate and unbound sample or standard are washed from the plate, and HRPStreptavidin (SABC) is added to each microplate well and incubated. Then TMB substrate solution is added to each well. The enzyme-substrate 
BAJOPAS Volume 13 Number 2, December, 2020

reaction is terminated by the addition of a sulphuric acid solution and the colour change is The concentration of target in the samples is then determined by comparing the OD of the samples to the standard curve and Testosterone were measured using an Enzyme Linked Immunosorbent Assay (ELISA) based assay technique. Further details available on the company website (www.fn-test.com).

The DEMEDITEC Testosterone rat/mouse ELISA Kit(obtained from Demeditec Diagnostics $\mathrm{GmbH}$, Kiel Germany) is a solid phase enzyme-linked immunosorbent assay(ELISA), based on the principle of competitive binding. An unknown amount of testosterone present in the sample and a defined amount of testosterone conjugated to horseradish peroxidase compete for the binding sites of testosterone antiserum coated to the wells of a microplate. After onehour incubation on a shaker the microplate is washed four times. After addition of the measured spectrophotometrically at a wavelength of 450nm. substrate solution the concentration of testosterone is inversely proportional to the optical density measured. Further details available on the company website (http://www.demeditec.com)

\section{Statistical Analysis}

Data obtained were analysed using SPSS (version 20.0). Mean values of all the assayed hormones in each group were calculated. Analysis of Variance was done to test for any significant differences among the groups. The level of significance was set as $p<0.05$.

\section{RESULTS}

The result showed no significant difference $(\mathrm{P}<$ $0.05)$ in the serum level of LH $(p=0.815)$, FSH $(p=0.783)$ and Testosterone $(p=0.330)$ between the control group (no soy diet) and the experiment groups (20\% and $50 \%$ soy diet).

Table 1: Effect of Dietary Supplementation with Soybean on Reproductive Hormones Levels

Hormone

$\mathrm{LH}(\mathrm{mIU} / \mathrm{ml}) \quad \mathrm{FSH}(\mathrm{mIU} / \mathrm{ml}) \quad$ Testosterone $(\mathrm{ng} / \mathrm{ml})$

\begin{tabular}{llll} 
Group & & & \\
\hline 1 & $0.92 \pm 0.18$ & $2.21 \pm 0.00$ & $7.83 \pm 0.00$ \\
2 & $0.89 \pm 0.27$ & $2.09 \pm 0.23$ & $6.95 \pm 0.77$ \\
3 & $0.90 \pm 0.46$ & $2.19 \pm 0.00$ & $7.73 \pm 0.01$ \\
ANOVA (F) & 0.206 & 0.247 & 1.155 \\
P-value & 0.815 & 0.783 & 0.330
\end{tabular}

Values as Mean \pm SD; Significance $=P<0.05$

\section{DISCUSSION}

The mean value of Follicle Stimulating Hormone in the control group of this study was $2.21 \pm 0.001$. A randomised study on effect of soybean on fertility of male and female albino rats (Serag El Din et. al., 2011) reported a mean value of Follicle Stimulating Hormone among the control group to be $4.69 \pm 0.23$. In the same study (Serag El Din et. al., 2011) the mean values of Follicle Stimulating Hormone among male albino rats fed on 30, 60 and $90 \mathrm{gm}$ cooked soybeans/70 kg human body weight (b.w.) for three months were $3.63 \pm 0.23$, $2.86 \pm 0.17$ and $2.60 \pm 0.38$ respectively which showed that the higher the soy bean content, the lower the mean hormone levels. In this study, the mean values of Follicle Stimulating Hormone among the Wistar rats fed on $20 \%$ and $50 \%$ soy diet was $2.09 \pm 0.23$ and $2.20 \pm 0.00$ respectively. This showed that from our study, the study group fed on $20 \%$ soy diet had a lower mean value of Follicle Stimulating Hormone when compared to the groups that were fed on soy free diet and $50 \%$ soy diet. This is contrary to the findings in a similar study (Serag El Din et. al., 2011) where the mice fed on higher concentration of soy diet had lower mean Follicle Stimulating Hormone values. However, the mean value of Follicle Stimulating Hormone in that study was almost twice of that reported in this study. This may be due to the difference in the species of rats used in the studies. Even though differences were observed in the mean values of Follicle Stimulating Hormone among the different study groups from our study, these differences were not statistically significant (Table 1). This finding agrees with the findings of Moderasi et al (Moderasi et. al., 2011) but is contrary to other findings in the literature where soybean was reported to cause a significant decrease in the levels of Follicle Stimulating Hormone.

The mean value of Luteinising Hormone among the control group in this study is $0.92 \pm 0.18$, whereas that of the groups that had $20 \%$ and $50 \%$ soybean was $0.89 \pm 0.27$ and $0.90 \pm 0.47$ respectively. This showed that the study group that was fed on $20 \%$ soy diet had the lowest mean Luteinising Hormone levels. In a similar study ${ }^{6}$ the levels of Luteinising Hormone among male albino rats fed on $30 \mathrm{~g} / 70 \mathrm{~kg}$ body weight, $60 \mathrm{~g} / \mathrm{kg}$ body weight and $90 \mathrm{~g} / \mathrm{kg}$ body weight 
BAJOPAS Volume 13 Number 2, December, 2020

were $0.74 \pm 0.14,0.64 \pm 0.05$ and $0.54 \pm 0.0 .06$ respectively. The mean value of Luteinising This showed that the higher the dietary soybean content, the lower the mean Luteinising Hormone levels with the control group having the highest mean value. The mean values obtained in this study are slightly higher than that obtained by the study on albino rats. Moderasi et al reported lower values among those fed on low soy diet (Moderasi et al., 2011). Their finding of lower mean value of Luteinising Hormones among rats fed on $20 \%$ soy diet is similar to the finding in this study. However, they reported a higher value of Luteinising Hormone among experimental groups that were fed on higher concentration of soy diet. Even though differences were observed in the mean values of Luteinising Hormone among the different study groups from our study, these differences were not statistically significant. This finding agrees with the findings of Moderasi et al were they reported that there was no significant difference in mean values of Luteinising hormone between rats fed on soy free diet when compared to rats fed $20 \%$ soy diet but contrary to their other findings that reported a significant increase in serum Luteinising Hormone levels among rats fed on high concentration ( $30 \%$ and $50 \%$ ) of soy diet when compared to control group. Soybean has also been reported to cause a significant decrease in values of Luteinising Hormone by other authors (Serag El Din et. al., 2011).

The mean level of Testosterone among the control group in this study was $7.83 \pm 0.00$ while those in the $20 \%$ soy diet and $50 \%$ soy diet were $6.96 \pm 0.77$ and $7.73 \pm 0.01$ respectively. This showed that the group fed on $20 \%$ soy diet had lower mean Testosterone level when compared to the groups that had soy free diet and $50 \%$ soy diet. Lower mean values of Testosterone due to ingestion of soy diets have been reported in both human and animal studies (Moderasi et al., 2011; Serag El Din et. al., 2011). A similar study (Moderasi et al., 2011) reported a lower value of Testosterone among rats fed on $20 \%$ soy diet as was observed in this study but higher values were recorded with higher concentration (50\%) of soy diet which is in contrast to our finding. In another human study (Goodin et al., 2007)ingestion of soy diet led to a significant decrease in serum concentration of testosterone, however, the effect was reversed within two weeks of stoppage (Goodin et al., 2007). Even though differences were observed in the mean values of Testosterone among the different study groups from our study these differences were not statistically significant. This is contrary to other
Hormone among the control group of the same study was $1.16 \pm 0.08$. findings in the literature where $20 \%$ soy diet was said to significantly decrease the serum testosterone values among rats when compared to control while $30 \%$ and $50 \%$ soy diets were said to significantly increase the serum testosterone values among rats when compared to control (Moderasi et al., 2011).

The findings in this research work with regards to the effect of dietary intake of soybean on serum testosterone is in keeping with studies reported by some authors in both human and animal experiments (Liang et. al., 2001; Perry et. al.,2007). Perry et al conducted an animal study on Cynomolgus monkeys over a period of three months (Perry et. al., 2007). The control group were fed soy free diet while the experiments group were fed soy-based diet. They concluded that there was no significant difference in serum levels of testosterone and sperm counts in all study groups. The researchers also confirmed presence of phytoestrogen in serum of experiment animals during the period of research. A Meta-analysis involving 32 reports involving 36 treatment groups in humans also reported that soy food and supplements do not alter the bioavailability of testosterone in men(Hamilton-reeves et. al., 2007).

Even though, at molecular level, soybean phytoestrogens have the ability to inhibit enzymes involved in steroidogenesis leading to decrease in testosterone resulting in a negative feedback on Gonadotrophin Releasing Hormone (GnRH) with subsequent cessation of production of $\mathrm{FSH}$ and $\mathrm{LH}$, this effect was not evident in this research work.

A direct explanation may not be evident. However, some factors may play a role in this finding. The process of preparation of the soybean may reduce the phytoestrogen content of the soybean thus limiting its effect on the reproductive hormones. Also, Soybean contains some anti-nutrients within it which includes trypsin inhibitors, phytin, lectins, saponins and haemagglutinins (Serag El Din et. al., 2011) which are affected by the way soybean is processed before consumption. It may be possible that while processing the soybean for dietary consumption, the composition of the various nutrients and other substances is affected which may in turn affect the bioavailability of the phytoestrogen content of soybean. The effects of these anti-nutrients content in relation to reproductive functions in males may also affect the role dietary soybean plays in male reproductive function. The effect of dietary soybean may also be affected by a 
BAJOPAS Volume 13 Number 2, December, 2020 background abnormality in steroidogenesis. This may further be worsened by ingestion of soybean. Modaresi et al also suggested that males with metabolic syndrome or those who are overweight should take precautions using oestrogenic compounds such as soy bean since such compounds decrease the expression of oestrogen receptors on testes tissue, occupy the active site of these receptors and have a negative role on hypothalamic-pituitary-gonadal axis. However, these factors may not be evident from this study.

\section{CONCLUSION}

Dietary Supplementation with $20 \%$ and $50 \%$ soybean did not cause significant alteration in the serum FSH, $\mathrm{LH}$ and Testosterone levels suggesting that soybean does not disrupt reproductive function in male Wistar rats.

\section{RECOMMENDATION}

1. It is also recommended that the effect of methods of processing soybean on the phytoestrogen content of soybean be studied to determine whether it affects the overall effect on male reproductive hormones. Also, the role of the other contents of soybean (trypsin inhibitors,

\section{REFERENCES}

Adlercneutz, H., \&Mazzur, W. (1997). Phytoestrogens and Western diet.Ann Med, 29, 95-120.

Adlercneutz, H., Mazzur, W., \& Bartels, P. (2000). Phytoestrogens and prostate disease. J Nutr, 130, 6585-95.

Atanassoun, N., McKinnell, C., Turner, K. J., Walker, M., Fisher, J. S., Morley, M., Millar, M. R., Groome, N. P., \& Sharpe, R. M. (2000). Comparative effects of neonatal exposure of male rats to potent and weak environmental oestrogens on spermatogenesis at puberty and the relationship to adult testis size and fertility: evidence for stimulatory effects of low estrogen levels. Endocrinology, 141(10), 3898-3907.

Crouse, J., Morgan, T., \& Terry, J. (1999). A randomised trial comparing effect of casein with that of soy protein containing varying amounts of isoflavones on plasma concentration of lipids and lipoproteins. Arch Intern Med, 159, 2070-2076.

Dillingham, B. L., McVeigh, B. L., Lampe, J. W., \& Duncan, A. M. (2005). Nutrition and Cancer Soy Protein Isolates of Varying Isoflavone Content Exert Minor Effects on Serum Reproductive Hormones in phytin, lectins, saponins and haemagglutinins) on reproductive function may shed more light on the association between soybean and male reproductive dysfunction. This is particularly important especially in our environment where soybean provides a rich source of cheap protein and has rightly been named the Cinderella crop of Africa.

2. Future research on the effect of dietary soybean on sperm quality and subsequent conception is also recommended.

3. Need for robust human intervention studies.

\section{LIMITATION}

The major limitation of this study is that it is a translational study and as such may not be directly applicable to humans. The research was time bound using personally financed resources which limited its scope.

AUTHOR CONTRIBUTION STATEMENT

All authors contributed to the conceptualization, study design, analysis, result discussion and final manuscript.

CONFLICT OF INTEREST

The Authors declare that no conflicting interests exist.

Healthy Young Men. J Nutr, 13, 5841591.

Fraser, L. R., Beyret, E., Milligan, S. R., \&Adeoya-Osiguwa, S.A. (2006). Effects of estrogenic xenobiotics on human and mouse spermatozoa. Hum Reprod, 21(5), 1184-1193.

Goodin, S., Shen, F., Shih, W. J., Dave, N., Kane, M. P., \& Medina, P. (2007). Clinical and Biological Activity of Soy Protein Powder Supplementation in Healthy Male Volunteers. Cancer Epidermiol Biomarkers Prev, 2007, 16, 1829-1834.

Hamilton-reeves, J. M., Goodin, S., Shih, W. J., \&Gallo, M. (2007).Effect of Soy Protein on Testosterone Levels. Cancer Epidemiology Biomarkers and Prevention, 16, 2796.

Knight, D., \& Eden, J. (1996). A review of the clinical effects of phytoestrogens. Obstet Gynecol, 87, 897-904.

Liang, Y., Davis, S., \& McGrath, B. P. (2001). Dietary Soy Has both Beneficial and Potentially Adverse Cardiovascular Effects: A Placebo-Controlled Study in Men and Postmenopausal Women. The Journal of clinical endocrinology $R$ metabolism. 86(7), 17-19. 
BAJOPAS Volume 13 Number 2, December, 2020

Lund, T. D., Munson, D. J., Haldy, M.E., Setchell, K. D. R., Lephart, E. D., \& Handa, R. J. (2004). Equol Is a Novel Anti-Androgen that Inhibits Prostate Growth and Hormone Feedback. Biology of Reproduction, 70, 1188-1195.

Messina, M., Nagata, C., \& Wu, A. H. (2006). Estimated Asian adult soy protein and isoflavone intakes. Nutr. Cancer, 55, 112.

Moderasi, M., Messripour, M., \&Khorami, $H$. (2011). Effect of soybean on male reproductive physiology in mice. IPCBEE, 3, 15-18.

Perry, D. L., Spedick, J. M., McCoy, T. P., Adams, M. R., Franke, A., \& Cline, J. M. (2007). Dietary soy protein containing isoflavonoids does not adversely affect the reproductive tract of male cynomolgus macaques (Macacafascicularis). J Nutr 137(6), 1390-1394.
Price, K., \& Fenwick, G. (1985). Naturally occurring oestrogens in foods-A review. Food AdditContam, 2(2), 73-106.

Serag El Din, O. S., Batta, H., A., \& Abd El Fattah, N. (2011). Effect of soybean on fertility of male and female albino rats. $J$ Am Sci, 7(6), 872-883.

Setchell, K. D. (1998). Phytogens: The biochemistry, physiology and implications for human health of soy isoflavones. $A m \mathrm{~J}$ Clin Nutr, 68(6), 1333S-1346S.

Song, T., Hendrich, S., \& Murphy, P. (1999). Estrogenic activity of glycitein, a soy isoflavone. J Agric Food Chem, 47, 1607-1610.

Weber, K. S., Setchell, K. D. R., Stocco, D. M., Lephart, E. D. (2001). Dietary soyphytoestrogens decrease testosterone levels and prostate weight without altering LH, Prostate 5 a-Reductase or Testicular Steroidogenic Acute Regulatory Peptide Levels in Adult Male Sprague-Dawley Rats. J Endocrinol, 170(3), 591-9. 